\title{
Indications for imaging studies in SARS-CoV-2 infected patients - recommendations of the Polish Medical Society of Radiology
}

\author{
Andrzej Cieszanowski ${ }^{1,2, A, B, D, D, E, F}$, Elżbieta Czekajska ${ }^{3, A, B, D, E, F}$, Mariusz Furmanek ${ }^{4, B, D, E, F}$, Barbara Giżycka ${ }^{5, A, B, D, E, E, F}$, \\ Katarzyna Gruszczyńska ${ }^{6, A, B, D, E, F}$, Joanna Podgórska ${ }^{1, A, B, D, E, F}$, Agnieszka Oronowicz-Jaśkowiak ${ }^{1, B, C, D, E, F,}$, \\ Zbigniew Serafin ${ }^{7, A, B, D, D, F}$, Edyta Szurowska ${ }^{8, A, B, D, E, F}$, Jerzy Walecki ${ }^{4, A, B, D, E, F}$
}

\author{
'Department of Radiology I, Maria Sklodowska-Curie National Research Institute of Oncology, Warsaw, Poland \\ ${ }^{2} 2^{\text {nd }}$ Department of Clinical Radiology, Medical University of Warsaw, Warsaw, Poland \\ ${ }^{3}$ Department of Radiology, Medical University of Lublin, Lublin, Poland \\ ${ }^{4}$ Department of Diagnostic Imaging, Centre of Postgraduate Medical Education, Warsaw, Poland \\ ${ }^{5}$ Department of Diagnostic Imaging, Multispeciality Hospital Megrez Sp. z 0.0., Tychy, Poland \\ ${ }^{6}$ Department of Radiology and Nuclear Medicine, Faculty of Medical Science in Katowice, Medical University of Silesia, Poland \\ ${ }^{7}$ Department of Radiology and Diagnostic Imaging, Nicolaus Copernicus University, Collegium Medicum, Bydgoszcz, Poland \\ ${ }^{8} 2^{\text {nd }}$ Department of Radiology, Medical University of Gdansk, Poland
}

\section{Abstract}

The pandemic involving COVID-19 caused by the SARS-CoV-2 coronavirus, due to its severe symptoms and high transmission rate, has gone on to pose a control challenge for healthcare systems all around the world. We present the third version of the recommendations of the Polish Medical Society of Radiology (PMSR), presuming that our knowledge on COVID-19 will advance further rapidly, to the extent that further supplementation and modification will prove necessary. These recommendations involve rules of conduct, procedures, and safety measures that should be introduced in radiology departments, as well as indications for imaging studies.

Key words: COVID-19, SARS-CoV-2, PMSR, PLTR.

\section{Introduction}

The pandemic involving the novel coronavirus disease 2019 (COVID-19) had its roots in Wuhan, Middle China, Hubei province, in November 2019. The first case in Europe was confirmed on $24^{\text {th }}$ January 2020; on $11^{\text {th }}$ March 2020, the World Health Organization (WHO) declared the COVID-19 pandemic [1]. In Poland, the first case was observed on $4^{\text {th }}$ March 2020. Over a year of global experience in prevention, diagnosis and treatment of the disease, as well as the appearance of many scientific publications on COVID-19, prompted the authors to update the recommendations of the Polish Medical Society of Radiology.
At the same time, despite unprecedented advances in many medical fields, including epidemiology, vaccinology, and radiology, the pandemic continues to pose a serious challenge to humanity, in particular due to new, more virulent strains that keep emerging in many countries, including Belgium, the UK, and Brazil [2].

The current diagnostic criteria for COVID-19 are based on clinical symptoms, epidemiological history (until local transmission in a given area has been documented), and laboratory tests. The final diagnosis is established based on the RT-PCR assay [3,4]. Although diagnostic criteria for COVID-19 do not include imaging [5], such as a radiograph (X-ray) or computed tomography (CT) of the

Correspondence address:

Agnieszka Sonia Oronowicz-Jaśkowiak, Department of Radiology I, Maria Sklodowska-Curie National Research Institute of Oncology, 15B Wawelska St., 00-001 Warsaw, Poland, e-mail: asjaskowiak@icloud.com

Authors' contribution:

A Study design · B Data collection · C Statistical analysis · D Data interpretation - E Manuscript preparation · F Literature search · G Funds collection 
chest, more and more scales and tools for radiologists are being developed to facilitate the diagnosis and assessment of the severity of the disease [6].

When considering ordering imaging, it should be remembered that X-ray and CT findings in patients with COVID-19 are non-specific and do not allow COVID-19 to be distinguished from other types of pneumonia [7]. Therefore, imaging should not be performed to confirm or rule out the diagnosis. Instead, it is used for the initial radiological assessment of the patient and for monitoring the evolution of the disease. Imaging should only be ordered in cases in which the results may have an impact on further management. At the same time, viral exposure to other patients and staff should be kept to a minimum.

\section{General guidelines for imaging in patients with suspected or confirmed COVID-19}

1. Based on imaging studies, SARS-CoV-2 infection cannot be diagnosed, but its pulmonary manifestations can be visualized [7].

2. Imaging should only be ordered in cases in which the results may have an impact on further management.

3. Dedicated imaging departments for SARS-CoV-2 positive patients are located in infectious disease centers or dedicated homonymous hospitals according to the Regulation of the Minister of Health [8].

4. In multidisciplinary hospitals with separate COVID-19 departments, a time window for all planned imaging studies is set in which patients with COVID-19 are examined, after which the rooms are decontaminated. In cases when urgent diagnostics in needed, i.e. outside the working hours of the radiology department, the patients are examined in the order dictated by the urgency of the case, and decontamination is performed after every examination in a SARS-CoV-2-positive patient.

5. Before the patient is transported to the radiology department, it is necessary to perform a SARS-CoV-2 test. Although PCR remains the gold standard [3], in many cases, due to time constraints, an antigen test is performed initially.

6. The referral must contain clear information on the suspected/confirmed COVID-19. This will make it possible to ensure adequate protection for the employees and patients of the radiology department. In hospitalized patients, it may be useful to include the CRP level and white blood cell count on the referral, as COVID-19 patients tend to have a high CRP and low WBC [9].

7. In any case of suspected COVID-19 infection, radiology department personnel must be provided with full personal protective equipment (PPE) (according to the Polish Medical Society of Radiology guidelines).

\section{Personal protective equipment for staff in direct contact with patients with suspected or confirmed COVID-19 infection}

The staff in direct contact with patients with suspected or confirmed COVID-19 infection (most commonly including radiographers, physicians during ultrasound examination, nurses, paramedics, cleaners) should be wearing PPE (Table 1). During the examination, the patient should wear a surgical mask.

Personnel should be trained in proper PPE use (infections often occur as PPE is removed) [13].

\section{Imaging modalities}

\section{Chest X-ray}

If the lunglesions are mild, the chest X-ray (CXR) may yield false-negative results [14].

\section{Indications:}

1. It is not recommended to use CXR as a screening tool for SARS-CoV-2 infection. The sensitivity and specificity of CXR in detecting infections in asymptomatic patients are low [15]. In the event of a high epidemiological burden of the region, it is recommended that, in the case of CXRs performed for other reasons, SARS-CoV-2 infection be included in the differential diagnosis of incidental findings in the chest.

2. Patients with no symptoms or mild symptoms (fever, cough, mild dyspnea) do not require imaging studies.

3. If lung assessment is clinically indicated (persistent cough and/or symptoms suggesting pulmonary involvement), CXR may be performed as a first-line imaging study. Despite the low CXR specificity, it may be helpful in making an initial diagnosis of COVID-19 when it is used together with a comprehensive clinical evaluation. The final diagnosis requires a positive RT-PCR test, which remains the gold standard $[3,4]$. In line with WHO recommendations [16], a negative RT-PCR test may be supplemented

Table 1. PPE for contact with patient with suspected or confirmed COVID-19 [10-12]

\begin{tabular}{|c|c|c|}
\hline Optimal equipment & $\begin{array}{l}\text { Acceptable equipment } \\
\text { (in the absence of optimal equipment) }\end{array}$ & $\begin{array}{l}\text { Equipment when contact is with } \\
\text { intubated patients }\end{array}$ \\
\hline $\begin{array}{l}\text { Dedicated waterproof/impermeable gown } \\
\text { Goggles } \\
\text { FFP2/3 respirator } \\
2 \text { pairs of gloves }\end{array}$ & $\begin{array}{l}\text { Nonwoven surgical head cap } \\
\text { Nonwoven gown + plastic surgery gown (on top) } \\
\text { Face shield } \\
\text { FFP2/3 respirator } \\
2 \text { pairs of gloves }\end{array}$ & $\begin{array}{l}\text { Nonwoven surgical head cap } \\
\text { Nonwoven gown } \\
\text { Face shield } \\
\text { Surgical mask } \\
2 \text { pairs of gloves }\end{array}$ \\
\hline
\end{tabular}


with imaging studies in COVID-19-suspected patients. It should be noted that the sensitivity of CXR for SARSCoV-2 diagnosis is $64 \%$, and specificity is $82 \%$ [16]. Therefore, the possibility of false-negative results at the onset of the disease should be taken into account.

4. There have been reports of SARS-CoV-2 mutations that yield a negative result in the commercially available RT-PCR test [17]. It is suggested that in the event of a clinical suspicion of COVID-19 and a negative test result, CXR should be performed due to the high specificity of the method [16].

5. Follow-up CXR in both stable and clinically unstable patients should be avoided, unless this is required to monitor progress of the disease and will impact upon patient management $[14,18]$

6. In critically ill patients with acute respiratory distress syndrome (ARDS), particularly in mechanically ventilated patients, CXR (using a portable X-ray machine) is the preferred lung imaging method [19].

\section{Examination procedure:}

It is advised to use portable X-ray machines for CXRs, as, in the vast majority of cases, it is sufficient to assess the lungs and makes it possible to avoid the transport of the patient within the hospital [20]. Optimally, these machines should remain where patients with COVID-19 are treated [11]. The examining radiographer puts on PPE before entering the patient's room. Having performed the examination, the radiographer removes one pair of gloves before disinfecting the machine with disinfecting wipes. The radiographer takes the machine out of the room before disinfecting it a second time [10]. Detailed guidelines are provided in a separate document: https:// pltr.pl/wp-content/uploads/2020/03/COVID-19-zasadypost\%C4\%99powania-zalecenia-PLTR-i-KK.pdf

\section{Chest computed tomography}

Chest CT may fail to show any abnormalities in the early stages of SARS-CoV-2 infection. While chest CT proves highly sensitive in detecting lung lesions, its specificity is very low [16]. Similar findings may therefore be made in patients with other types of pneumonia, including that caused by other viruses (H1N1 influenza, adenovirus, CMV) or fungi (pneumocystosis caused by Pneumocystis jiroveci or P. carinii), COP (cryptogenic organizing pneumonia), drug-induced pulmonary toxicity, hypersensitivity, and autoimmune disease [21].

\section{Indications:}

It is not recommended to use chest $\mathrm{CT}$ as a screening tool for SARS-CoV-2 infection. The sensitivity and specificity of $\mathrm{CT}$ in detecting infections in asymptomatic patients are low [22]. In the event of a high epidemiological burden of the region, it is recommended that, in the case of CTs performed for other reasons, SARS-CoV-2 infection be included in the differential diagnosis of incidental findings in the chest.
A CT scan should only be ordered in a SARS-CoV-2positive patient if the result will impact upon patient management, in particular to estimate lung involvement or exclude thromboembolic complications [14,18]. Benefits and risks (including patient transport through the hospital, necessity to secure the route of transport, CT machine decontamination, lower availability of CT scans for other patients) of the examination should therefore be considered before a CT scan is ordered [14].

Chest CT may be performed for baseline evaluation of lungs in patients hospitalized for COVID-19, in cases when it may facilitate monitoring of disease progression. There are several diagnostic scales for estimating the probability of SARS-CoV-2 infection, such as the CO-RADS scale [23]. It is recommended to use structured reports, including the estimated percentage of pulmonary involvement, both in general and with the division into ground glass opacity and consolidations. In centers that have access to artificial intelligence algorithms, it is advisable to validate them and apply them for lung involvement assessment, taking into account the volume of spared lung parenchyma.

In selected cases (e.g. where the waiting time for an RT-PCR test is long, no antigen tests are available, a false negative RT-PCR is suspected, or clinical symptoms suggest COVID-19), a clinician may, along with a radiologist, consider a CT scan - if this will impact upon patient management [14]. While non-specific, a CT image may assist with the initial diagnosis of COVID-19 [14], even if the final diagnosis requires RT-PCR confirmation $[18,24]$. The accuracy of CT in SARS-CoV-2 diagnosis should be taken into account - when compared with RT-PCR, the sensitivity of CT is $87 \%$, specificity $46 \%$, and positive predictive value $89 \%$ [25]. The possibility of false-negative results at the onset of the disease should be taken into account [14].

In both stable and unstable disease (Modified Early Warning Score - MEWS $<3$ points or 3-4 points, respectively), CT is highly sensitive at detecting interstitial changes and consolidations, as well as assessing their dynamics $[14,24,26]$. Moreover, along with acid-base balance, it is of predictive value $[26,27]$.

CXR remains the method of choice in patients in a critical condition with ARDS, other organ failure (hypotension, shock, multi-organ failure; MEWS $>4$ points), and in mechanically ventilated patients $[18,19]$. In selected cases where $\mathrm{CT}$ is required, the patient should be connected to a mobile ventilator to minimize the risk of infection for CT staff [18].

Chest CT is also indicated in patients with suspected complications, such as empyema or lung abscess, as well as in patients with suspected co-existing conditions such as pulmonary embolism $[14,18]$.

Routine follow-up chest CT of recovered patients is not recommended. In special circumstances, follow-up examination may be useful in patients who have suffered from severe COVID-19 or have concomitant chronic lung disease. A follow-up may help identify patients who 
have developed pulmonary fibrosis after a severe form of COVID-19 [28].

\section{Examination procedure:}

Routinely, a CT scan for COVID-19 is performed with no intravenous contrast administered [14].

The examination technique depends on the machine and protocols used in a given center.

High-resolution CT (HRCT) is recommended wherever possible [29].

Contrast-enhanced CT is performed in certain situations, including where it is suspected that patients with COVID-19 have concomitant diseases/complications (e.g. suspected pulmonary embolism) $[14,18]$.

Detailed guidelines are provided in a separate document: https://pltr.pl/wp-content/uploads/2020/03/ COVID-19-zasady-post\%C4\%99powania-zalecenia-PLTRi-KK.pdf.

\section{Chest ultrasound}

Lung ultrasound examination and interpretation of the obtained images require extensive experience, as well as close and long (up to 30 minutes) contact between the physician and the patient, which increases the risk of infection for the physician. It is not recommended for physicians without sufficient experience in the field to perform lung ultrasound. The pandemic is also not the time to train physicians in this area, as it unnecessarily exposes them to infection.

Lung ultrasound is not on the training curriculum for radiology residents in Poland and has only been used rarely so far (mainly in children attending highly specialized centers, with a view to exposure to ionizing radiation being minimized). Thus a majority of radiologists do not have the necessary experience to perform this examination. In addition, there are only a few reports confirming the effectiveness of ultrasound in diagnosing COVID-19 [30]. Therefore, ultrasound may not be used to diagnose COVID-19, and may only confirm the presence of lesions in peripheral parts of lungs. In selected cases, ultrasound can be used to monitor the course of the disease.

Pleural ultrasound to visualize effusion should not be confounded with lung ultrasound. However, given the need for only basic experience with ultrasonography, it is easy to diagnose pleural effusion (a symptom seen rarely in COVID-19 patients [31], especially in the early stages of the disease) [32].

\section{Indications:}

Monitoring peripheral pulmonary lesions in patients with respiratory insufficiency - but only by a team experienced in lung ultrasound.

\section{Examination procedure:}

The examination extends to the entire available chest area.

The probe is applied in standardized areas specified in the guidelines for lung and pleural ultrasound.
In ventilated patients in a critical condition, the examination is most often limited to the anterior and lateral areas.

In patients in the prone position, the examination involves posterior and lateral areas of the chest.

It is typical for a convex probe to be used.

A linear probe may also be deployed, to assess the pleural line and detect consolidations.

The probe is applied perpendicular to the ribs so at least two adjacent ribs and the pleural line between them are visualized.

\section{Echocardiography}

Echocardiography is indicated in patients with COVID-19 suffering from suspected acute heart failure in the course of respiratory failure [33].

\section{Chest magnetic resonance imaging}

\section{Indications:}

Although chest MRI does not play a significant role in COVID-19 diagnosis, it is increasingly used in the diagnosis of its complications. The American College of Radiology recommends limiting the use of MRI to strictly necessary cases and postponing all scheduled examinations [34].

Neurological complications - the most common ones include hemorrhagic and ischemic stroke, cerebral venous sinus thrombosis, meningeal enhancement, encephalomyelitis, and diffuse, hyperintense areas in white matter, which may be associated with recurring microhemorrhages [35]. In most cases, it is not possible to determine whether the findings are related to SARS-CoV-2 infection or are the result of comorbidities.

The incidence of cardiovascular complications in COVID-19 patients has not been investigated. It is possible that the heart is damaged during the disease, for example secondary to myocarditis [37]. According to a study published several months after the onset of the pandemic, elevated serum troponin was present in 36\% of patients infected with SARS-CoV-2 [40]. Some reports estimate that up to $7 \%$ of all deaths due to COVID-19 are caused by myocarditis [36]; however, these results are not confirmed by the presence of viral genetic material in cardiomyocytes or autopsy [41]. Imaging studies are not sufficient to diagnose myocarditis, and myocardial biopsy is currently not recommended in SARS-CoV-2 patients. Echocardiography remains the modality of choice [42]. If a patient develops clinical symptoms of myocarditis, current guidelines must be followed and MRI is frequently recommended [43].

\section{Examination procedure:}

It is believed that the use of face masks in patients with COVID-19 during MRI examination provides some degree of protection for other patients and staff [38]. It is 
recommended to provide MR safe masks as there have been reports of facial burns in patients examined on $3 \mathrm{~T}$ MRI scanners [39]. Replacing masks or ventilators with ones that are safe to use in the electromagnetic fields should take place before the patient's arrival at the MRI department. If such masks are not available, the metal parts of the mask or ventilator should be removed prior to the patient's arrival at the MRI department and the mask can be sealed using tape. It should be noted that the masks may contain other metal elements apart from the metal strip facilitating the adjustment to the face, such as woven metal fibers or nanoparticles that are intended to increase the antimicrobial properties of the product.

It must be verified that the PPE provided for the staff is MR safe.

MR scanner decontamination protocols vary widely between centers. General recommendations include decontamination of all visible surfaces, starting with those located higher and moving downwards [34].

\section{Summary}

1. Imaging studies allow lung lesions to be visualized, but do not enable COVID-19 diagnosis, which is based on a positive PCR test result.

2. Chest CT is of high sensitivity, but very low specificity, in diagnosing COVID-19.

3. Imaging studies (CXR, CT) should only be performed when the results can alter patient management.

4. CXR using a portable unit is recommended, on a point-of-care testing basis.

5. Routinely, non-contrast high-resolution chest CT (HRCT) is performed on COVID-19 patients.

6. Chest CT may be performed for initial/benchmark lung assessment in COVID-19 patients in cases where this may facilitate disease follow-up.

7. Contrast-enhanced chest CT is only performed when there is a suspicion of comorbid conditions, such as pulmonary embolism.

8. Clear information regarding suspected/confirmed COVID-19 is required on the referral, as better protection of RD staff and patients is then possible.

9. Staff of an RD examining a patient with suspected or confirmed COVID-19 infection must be provided with adequate PPE (in line with PMSR guidelines).

10. Lung ultrasound is a specialized examination not suitable for diagnosing COVID-19. This examination is only recommended for physicians with extensive experience in this field.

11. MRI is used for the diagnosis of COVID-19 complications (including neurological and cardiovascular complications).

\section{Conflict of interest}

The authors report no conflict of interest.

\section{References}

1. Huang C, Wang Y, Li X, et al. Clinical features of patients infected with 2019 novel coronavirus in Wuhan, China. Lancet 2020; 395: 497-506.

2. John G, Sahajpal NS, Mondal AK, et al. Next-Generation Sequencing (NGS) in COVID-19: a tool for SARS-CoV-2 diagnosis, monitoring new strains and phylodynamic modeling in molecular epidemiology. Curr Issues Mol Biol 2021; 43: 845-867.

3. World Health Organization. Coronavirus disease 2019 (COVID-19): situation report, 51. World Health Organization; 2020.

4. Zu ZY, Jiang MD, Xu PP, et al. Coronavirus Disease 2019 (COVID-19): a perspective from China. Radiology 2020; 296: E15-E25.

5. BSTI. COVID-19: BSTI Statement and Guidelines. [Internet]. Available from: https://www.bsti.org.uk/media/resources/files/BSTI_COVID-19_ Radiology_Guidance_version_2_16.03.20.pdf (Accessed: 27.03.2020).

6. Wasilewski P, Mruk B, Mazur S, et al. COVID-19 severity scoring systems in radiological imaging-a review. Pol J Radiol 2020; 85: e361-e368.

7. Kanne JP, Little BP, Chung JH, et al. Essentials for radiologists on COVID-19: an update - Radiology Scientific Expert Panel. Radiology 2000; 296: E113-E114.
8. Koronawirus: informacje i zalecenia. [Internet]. Available from: https:// www.gov.pl/web/koronawirus/lista-szpitali (Accessed: 10.11.2021).

9. Ferrari D, Motta A, Strollo M, et al. Routine blood tests as a potential diagnostic tool for COVID-19. Clin Chem Lab Med 2020; 58: 10951099.

10. Huang Z, Zhao S, Li Z, et al. The Battle Against Coronavirus Disease 2019 (COVID-19): Emergency Management and Infection Control in a Radiology Department [Internet]. [cited 27 March 2020]. Available from: https://www.unboundmedicine.com/medline/citation/32208140/ The_Battle_Against_Coronavirus_Disease_2019_(COVID-19):_Emergency_Management_and_Infection_Control_in_a_Radiology_Department.

11. World Health Organization. Infection prevention and control during health care when COVID-19 is suspected. Interim guidance [Internet]. 2020. Available from: https://apps.who.int/iris/bitstream/handle/10665/331495/WHO-2019-nCoV-IPC-2020.3-eng.pdf.

12. Lee SA, Grinshpun SA, Reponen T. Respiratory performance offered by N95 respirators and surgical masks: human subject evaluation with 
$\mathrm{NaCl}$ aerosol representing bacterial and viral particle size range. Ann Occup Hyg 2008; 52: 177-185.

13. Mitchell R, Roth V, Gravel D, et al. Are health care workers protected? An observational study of selection and removal of personal protective equipment in Canadian acute care hospitals. Am J Infect Control 2013; 41: $240-244$.

14. Mossa-Basha M, Meltzer CC, Kim DC, et al. Radiology Department preparedness for COVID-19: Radiology Scientific Expert Panel. Radiology 2020; 296: E106-E112.

15. Weinstock MB, Echenique ANA, Russel JW, et al. Chest X-ray findings in 636 ambulatory patients with COVID-19 presenting to an urgent care center: a normal chest $\mathrm{x}$-ray is no guarantee. J Urgent Care Med 2020; 14: 13-18.

16. Akl EA, Blažić I, Yaacoub S, et al. Use of chest imaging in the diagnosis and management of COVID-19: a WHO rapid advice guide. Radiology 2021; 298: E63-E69.

17. Jindal H, Jain S, Suvvari TK, et al. False-negative RT-PCR findings and double mutant variant as factors of an overwhelming second wave of COVID-19 in India: an emerging global health disaster. SN Compr Clin Med 2021; doi: 10.1007/s42399-021-01059-z.

18. ACR Recommendations for the use of Chest Radiography and Computed Tomography (CT) for Suspected COVID-19 Infection [Internet]. Available from: https://www.acr.org/Advocacy-and-Economics/ACR-Position-Statements/Recommendations-for-Chest-Radiography-and-CT-for-Suspected-COVID19-Infection (Accessed: 02.04.2020).

19. Jin YH, Cai L, Cheng ZS, et al. A rapid advice guideline for the diagnosis and treatment of 2019 novel coronavirus (2019-nCoV) infected pneumonia (standard version). Mil Med Res 2020; 7: 4.

20. Blažić I, Brkljačić B, Frija G. The use of imaging in COVID-19 - results of a global survey by the International Society of Radiology. Eur Radiol 2021; 31: 1185-1193.

21. Hani C, Trieu NH, Saab I, et al. COVID-19 pneumonia: a review of typical CT findings and differential diagnosis. Diagn Interv Imaging 2020; 101: 263-268.

22. Zeng Y, Fu J, Xu X, et al. Should computed tomography (CT) be used as a screening or follow-up tool for asymptomatic patients with SARSCoV-2 infection? Quant Imaging Med Surg 2020; 10: 1150-1152.

23. Prokop M, van Everdingen W, van Rees Veliinga T, et al. CO-RADS: a categorical CT assessment scheme for patients suspected of having COVID-19 - definition and evaluation. Radiology 2020; 296: E97-E104.

24. Fang Y, Zhang H, Xie J, et al. Sensitivity of chest CT for COVID-19: comparison to RT-PCR. Radiology 2020; 296: E115-E117.

25. Khatami F, Saatchi M, Zadeh SST, et al. A meta-analysis of accuracy and sensitivity of chest CT and RT-PCR in COVID-19 diagnosis. Sci Rep 2020; 10: 22402.

26. Song F, Shi N, Shan F, et al. Emerging 2019 novel coronavirus (2019nCoV) pneumonia. Radiology 2020; 295: 210-217.

27. Salehi S, Abedi A, Balakrishnan S, Gholamrezanezhad A. Coronavirus disease 2019 (COVID-19): a systematic review of imaging findings in 919 patients. AJR Am J Roentgenol 2020; 215: 87-93.

28. Han X, Fan Y, Alwalid O, et al. Six-month follow-up chest CT findings after severe COVID-19 pneumonia. Radiology 2021; 299: E177-E186.

29. Diao K, Han P, Pang T, et al. HRCT imaging features in representative imported cases of 2019 novel coronavirus pneumonia. Precis Clin Med 2020; 3: 9-13.
30. Ultrasonografia płuc u pacjentów z COVID-19 - praktyczny przewodnik dla lekarzy. Available from: https://criticalusg.org/2020/03/17/ ultrasonografia-pluc-u-pacjentow-z-covid-19-praktyczny-przewodnik-dla-lekarzy/?fbclid=IwAR3mMGmWDsefSusUKIIH08Jkfr327Hl9HJyr_Ha6ZHaQ2K3vmTRLcvmzRlo.

31. Shi H, Han X, Jiang N, et al. Radiological findings from 81 patients with COVID-19 pneumonia in Wuhan, China: a descriptive study. Lancet Infect Dis 2020; 20: 425-434.

32. Buonsenso D, Pata D, Chiaretti A. COVID-19 outbreak: less stethoscope, more ultrasound. Lancet Respir Med 2020; 8: e27.

33. Dweck M, Bularga A, Hahn RT, et al. Global evaluation of echocardiography in patients with COVID-19. Eur Heart J Cardiovasc Imaging 2020; 21: 949-958.

34. ACR guidance on COVID-19 and MR use. Available from: https:// www.acr.org/Clinical-Resources/Radiology-Safety/MR-Safety/COVID19-and-MR-Use.

35. Sheraton M, Deo N, Kashyap R, et al. A review of neurological complications of COVID-19. Cureus 2020; 12: e8192.

36. Ruan Q, Yang K, Wang W, et al. Clinical predictors of mortality due to COVID-19 based on an analysis of data of 150 patients from Wuhan, China. Intensive Care Med 2020; 46: 846-848.

37. Puntmann VO, Carerj ML, Wieters I, et al. Outcomes of cardiovascular magnetic resonance imaging in patients recently recovered from coronavirus disease 2019 (COVID-19). JAMA Cardiol 2020; 5: 1265-1273.

38. Lee SA, Grinshpun SA, Reponen T. Respiratory performance offered by N95 respirators and surgical masks: human subject evaluation with $\mathrm{NaCl}$ aerosol representing bacterial and viral particle size range. Ann Occup Hyg 2008; 52: 177-185.

39. Yee KM. FDA issues face mask warning for MRI exams. Available from: https://www.auntminnie.com/index.aspx?sec $=$ ser $\&$ sub $=-$ def\&pag=dis\&ItemID=131064.

40. Lala A, Johnson KW, Januzzi JL, et al. Prevalence and impact of myocardial injury in patients hospitalized with COVID-19 infection. J Am Coll Cardiol 2020; 76: 533-546.

41. Ozierański K, Tyminska A, Jonik S, et al. Clinically suspected myocarditis in the course of severe acute respiratory syndrome novel coronavirus-2 infection: fact or fiction? J Card Fail 2021; 27: 92-96.

42. Ferrer-Gómez A, Pian-Arias H, Carretero-Barrio I, et al. Late cardiac pathology in severe Covid-19. A postmortem series of 30 patients. Front Cardiovasc Med 2021; 8: 748396.

43. Ferreira VM, Schulz-Menger J, Holmvang G, et al. Cardiovascular magnetic resonance in nonischemic myocardial inflammation: expert recommendations. J Am Coll Cardiol 2018; 72: 3158-3176. 